\title{
c-Fos Induction in the Rat Nucleus of the Solitary Tract Correlates with the Retention and Forgetting of a Conditioned Taste Aversion
}

Thomas A. Houpt, ${ }^{1,2,3}$ Jennifer M. Philopena, ${ }^{1}$ Tong H. Joh, ${ }^{2}$ and

Gerard P. Smith ${ }^{1}$

${ }^{1}$ The E.W. Bourne Behavioral Research Laboratory

Department of Psychiatry

${ }^{2}$ Laboratory of Molecular Neurobiology

Burke Medical Research Institute

Department of Neurology and Neuroscience

Cornell University Medical College

White Plains, New York 10605

\begin{abstract}
Recently, we have described a potential neuronal correlate of the behavioral expression of a conditioned taste aversion (CTA) against sucrose at the level of c-Fos expression. Intraoral infusions of sucrose induce c-Fos-like immunoreactivity (c-FLI) in the intermediate nucleus of the solitary tract (iNTS) after a CTA has been acquired for sucrose. Sucrose infusions do not induce C-FLI in the iNTS of unconditioned rats or in conditioned rats after extinction of the CTA. Here, we describe persistence of altered responsiveness of the iNTS in rats with CTAs against sucrose by intraorally infusing sucrose 2 days, 3 months, or 6 months after acquisition of the CTA. Sucrose infusions induced c-FLI in the iNTS 6 months after conditioning. The behavioral expression of the CTA was attenuated at 6 months but not at 3 months; the number of c-FLI positive cells in the iNTS was proportional to the magnitude of the expression of the CTA. This evidence strengthens our hypothesis that c-FLI in the iNTS is a neuronal correlate of the expression of a CTA.
\end{abstract}

\footnotetext{
${ }^{3}$ Corresponding author.
}

\section{Introduction}

Conditioned taste aversion (CTA) is an unusual form of associative learning because the CTA to a taste paired previously with illness is retained for months or years, even after only one pairing of taste and illness (Seligman 1970). The time course of CTA retention is much longer than that seen in most other forms of associative learning in which conditioned reflexes are rarely retained beyond days or weeks even after dozens or hundreds of acquisition trials.

It has been demonstrated recently that intraorally infused sucrose (Houpt et al. 1994) or saccharin (Swank and Bernstein 1994) induces large numbers of c-Fos-like-immunoreactive cells in the intermediate nucleus of the solitary tract (iNTS) of rats with a previously acquired CTA. Sucrose or saccharin infusions do not induce c-Fos-like immunoreactivity (c-FLI) in the iNTS of unconditioned rats. Furthermore, sucrose does not induce c-FLI in the iNTS after the CTA is extinguished by presenting multiple infusions of sucrose without contingent toxic $\mathrm{LiCl}$ injections (Houpt et al. 1994). The induction of c-FLI in the iNTS by intraoral infusions of sucrose represents altered responsiveness of the iNTS to the taste stimulus after CTA acquisition, and, thus, c-FLI in the iNTS is a potential neuronal correlate of CTA expression.

To establish that c-FLI in the iNTS correlates with CTA expression under conditions other than

LEARNING \& MEMORY 3:25-30 (c) 1996 by Cold Spring Harbor Laboratory Press ISSN1072-0502/96 \$5.00

$$
\begin{aligned}
& \begin{array}{llllllllll}
L & E & A & R & N & I & N & G & \boldsymbol{Q}
\end{array} \\
& M \quad E \quad M O R \quad Y
\end{aligned}
$$


recent acquisition and active extinction, we examined the long-term retention and forgetting of a CTA acquired by pairing intraoral sucrose infusions and toxic $\mathrm{LiCl}$ injections. We processed the iNTS of rats for c-FLI after the intraoral sucrose infusions 2 days, 3 months, and 6 months after CTA acquisition to determine if altered c-FLI in the iNTS paralleled the long-term time course of behavioral expression of the CTA.

\section{Materials and Methods}

\section{ANIMALS AND SURGERY}

Adult male Sprague-Dawley rats ( $300 \mathrm{~g}$ ) were individually housed under a 12-hr light, 12-hr dark cycle at $25^{\circ} \mathrm{C}$. Ad lib food (Purina rodent chow) and water were provided except as noted below.

Anterior sublingual intraoral catheters were implanted under metofane anesthesia by a modified version of the technique described by Tyrka and Smith (1993). Intraoral catheters were prepared from $10 \mathrm{~cm}$ of $\mathrm{PE}-50$ polyethylene tubing; one end of the catheter was heat-flared to form a 2-mm-diameter annular end. A small incision was made on the ventral midline between the mandibles, and a bent 23-gauge syringe needle was pushed between the mandibles until the barrel projected into the mouth midway between the root of the lower incisors and the base of the tongue. The unflared end of the catheter was affixed to the end of the syringe needle; the needle was retracted to pull the tubing along the needle tract and out the incision on the ventral submental surface until the flared end of the catheter rested on the floor of the mouth beneath the tongue. An incision was then made from the caudal extent of the skull to midway between the scapulas on the dorsal surface of the rat's neck. A blunt wire probe was threaded between the skin and the musculature from the dorsal incision to the ventral submental incision. Then, the end of the intraoral catheter was attached to the wire probe, which was then pulled back with the intraoral catheter under the skin and externalized through the dorsal incision. The intraoral catheter was held in place by threading it through an outer sleeve of 0.040 silastic tubing attached to a 15 -mm-diameter. Marlex mesh disk (Bard-Parker) sutured to the dorsal neck musculature. A 5-cm length of sleeve and catheter projected from the dorsal surface of the rat for attachment to an infusion catheter. The dor- sal neck incision was closed with wound clips on either side of the catheter sleeve, and the submental incision was sutured closed.

\section{CTA FORMATION}

CTAs to intraoral infusions of $5 \%$ sucrose were formed in 12 rats by pairing intraoral sucrose infusions with $\mathrm{LiCl}$ injections. Conditioning was conducted on the rats in two groups of six over 2 weeks. Rats were deprived of food, but not water, for $17 \mathrm{hr}$ prior to intraoral infusions. Individual rats were weighed and placed in test chambers 26 $\mathrm{cm}$ wide by $17 \mathrm{~cm}$ deep by $30 \mathrm{~cm}$ tall (formed by subdividing a 40-gallon glass aquarium with Plexiglas walls). Syringe pumps (Harvard Apparatus) infused $5 \%$ sucrose from $20-\mathrm{ml}$ syringes at a rate of $1.1 \mathrm{ml} / \mathrm{min}$ for $6 \mathrm{~min}$ (setting 10) through 0.0040 gauge silastic catheters attached to the externalized end of the implanted intraoral catheters. Rats and any feces produced in the test chamber during the infusion were weighed immediately after the 6-min infusion; the weight gained during the infusion procedure was recorded as a measure of consumption of $5 \%$ sucrose. The rats were returned to their home cages and injected with $\mathrm{LiCl}(0.15 \mathrm{M}$, $12 \mathrm{ml} / \mathrm{kg}$, i.p.) $30 \mathrm{~min}$ after the start of the intraoral infusion. Intraoral sucrose infusions were paired with $\mathrm{LiCl}$ injections a total of three times at 48-hr intervals. All tests were conducted $2-6 \mathrm{hr}$ after lights on.

Three days after the third pairing of sucrose and $\mathrm{LiCl}$, the intraoral catheters were removed under metofane anesthesia. Intraoral catheters were reimplanted under metofane anesthesia in six of the rats 3 months after the third pairing of intraoral sucrose infusion and $\mathrm{LiCl}$. Three days after reimplantation, the rats were deprived of food for $17 \mathrm{hr}$ and received a final test intraoral infusion of $5 \%$ sucrose $(6.6 \mathrm{ml}$ over $6 \mathrm{~min})$. The rats were weighed immediately before and after the test infusion and returned to their home cages at the end of the infusion. One hour after the start of this sucrose infusion, the rats were sacrificed and processed for c-FLI as described below.

The remaining six rats were reimplanted with intraoral catheters 6 months after their third sucrose infusion and $\mathrm{LiCl}$ injection. The 6-month group also received an intraoral infusion of $5 \%$ sucrose (6.6. $\mathrm{ml}$ over $6 \mathrm{~min}$ ) $1 \mathrm{hr}$ before sacrifice and processing for c-FLI.

As a control for variability between the immu-

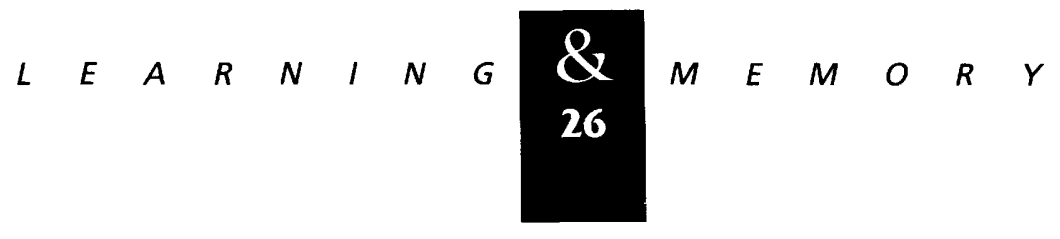


nohistochemical assays (which were separated by 3 months), control rats were tested, sacrificed, and processed in parallel on the same days as the 3and 6-month rats. The control rats were conditioned against sucrose by pairing an intraoral infusion of $5 \%$ sucrose with $\mathrm{LiCl}(0.15 \mathrm{M}, 12 \mathrm{ml} / \mathrm{kg}$, i.p.) three times, as above. The final pairing was conducted $72 \mathrm{hr}$ before the control rats were sacrificed on the same day as the 3-or 6-month rats. Thus, for the control rats, only 2 days intervened between the third sucrose- $\mathrm{LiCl}$ pairing and the final sucrose test and sacrifice. Three 2-day control rats were sacrificed and processed on the same days as the 3-month rats, and three 2-day control rats were sacrificed and processed on the same days as the 6-month rats.

\section{TISSUE COLLECTION AND}

\section{IMMUNOHISTOCHEMISTRY}

Rats were overdosed with sodium pentobarbital and, when completely unresponsive, transcardially perfused first with $100 \mathrm{ml}$ of heparinized isotonic saline containing $0.5 \% \mathrm{NaNO}_{2}$, then with $400 \mathrm{ml}$ of $4 \%$ paraformaldehyde in $0.1 \mathrm{M}$ sodium phosphate buffer (PB). The brains were dissected, blocked, postfixed for $2 \mathrm{hr}$, and transferred into $30 \%$ sucrose for cryoprotection. Forty-micron coronal sections were cut on a freezing, sliding microtome through the rostral-caudal extent of the NTS. Thirty-six sections were cut from the caudal subpostremal NTS (bregma, $14 \mathrm{~mm}$ ) to caudal extent of the rostral NTS [bregma, $12.8 \mathrm{~mm}$; all coordinates based on Paxinos and Watson (1986)]. Every other section collected from the NTS was processed for c-FLI.

Free-floating tissue sections were washed twice for $15 \mathrm{~min}$ in $0.1 \mathrm{M}$ sodium phosphate-buffered saline (PBS), then permeabilized in $0.2 \%$ Triton, $1 \%$ bovine serum albumin (BSA) in PBS for 30 min. After washing twice in PBS-BSA, sections were incubated overnight with a sheep anti-c-Fos peptide antibody (Cambridge Research Biochemicals) at a dilution of 1:3000. Because this antibody was raised against a peptide sequence common to other Fos-family members, it may cross-react with other proteins thus visualizing c-FLI (as opposed to specific c-Fos immunoreactivity). Sections were washed in PBS-BSA twice and incubated for $1 \mathrm{hr}$ with a biotinylated anti-sheep rabbit antibody (Vector Laboratories); bound secondary antibody was then amplified with the Vector Elite ABC kit.
Antibody complexes were visualized by a 5 -min, $0.5 \%$ diaminobenzidine reaction.

Cells expressing positive, nuclear c-FLI were quantified in the nucleus of the solitary tract (NTS) medial to the solitary tract. Counts were made by eye at $50 \times$ magnification and were made blind to the condition of individual rats. Only dark (nearly black), punctate nuclear staining was counted; diffusely stained cell bodies were not counted, nor were very faintly stained (light brown) nuclei counted. The NTS was divided into three subregions: caudal [ventral and caudal to the area postrema (AP)], intermediate (abutting the fourth ventricle), and rostral (where the NTS separates from the fourth ventricle). Each of these three subregions was represented by $\sim 6$ sections of the 18 NTS sections processed from each rat. Cell counts for all sections within each subregion of each rat were averaged, and the individual mean counts for each region were averaged across rats by subregion within experimental groups. All data were analyzed by analysis of variance; subsequent analyses were performed using Fisher's post hoc test.

\section{Results}

\section{SUCROSE INTAKE AND BEHAVIOR}

Pairing toxic $\mathrm{LiCl}$ injections with intraoral sucrose infusions sharply reduced intake of intraorally delivered sucrose over the course of conditioning (Fig. 1). All rats consumed most, if not all, of their initial infusion of $5 \%$ sucrose, which preceded the first injection of $\mathrm{LiCl}$ by $30 \mathrm{~min}$. The second infusion of sucrose was administered $48 \mathrm{hr}$ after the first pairing of sucrose and $\mathrm{LiCl}$; intakes were reduced to $50 \%$ or less of total consumed during the first infusion. By the third pairing, intakes were close to zero. Except for a small, but significant, difference in intakes between the 3 -month group and the 2-day group during the third infusion, all three groups had very similar reductions in intraoral sucrose intake and identical changes in behavioral response during the conditioning procedure.

The decline in intakes was accompanied by a change in orofacial movements and behavioral responses to the sucrose infusion. During the first infusion, the rats displayed typical ingestive (palatable) responses, such as mouth movements and tongue protrusions. During the second and third

$$
\text { …… }
$$




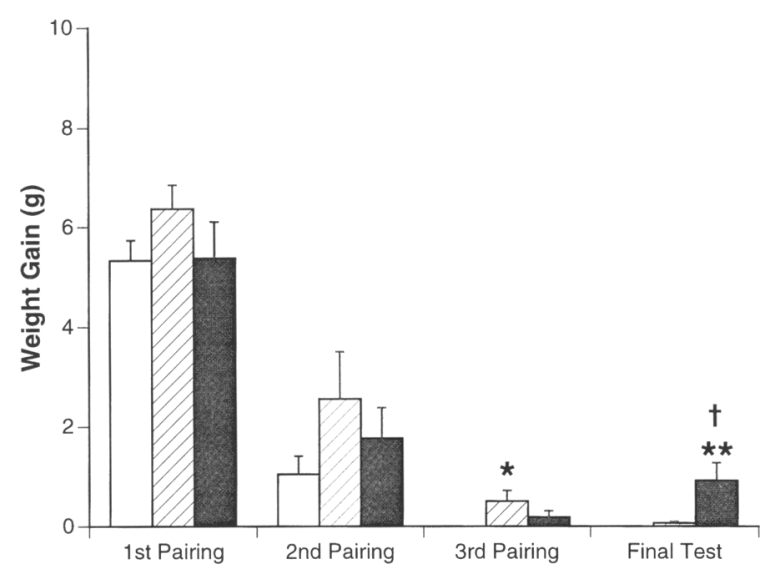

Figure 1: Weight gained during intraoral infusions of $5 \%$ sucrose $(6.6 \mathrm{ml}$ over $6 \mathrm{~min})$ during three pairings with $\mathrm{LiCl}(0.15 \mathrm{M}, 12 \mathrm{ml} / \mathrm{kg}$ i.p. injected $30 \mathrm{~min}$ after start of infusion) and during a final test infusion administered 2 days (open bars), 3 months (hatched bars), or 6 months (solid bars) after the third pairing. (Asterisk) $(P)<0.05$; (double asterisk) $P<0.005$ significantly different from 2-day group; (dagger) $P<0.05$ significantly different from 3-month group. $n=6$ in each group.

infusions, these ingestive responses were replaced by aversive responses, such as forearm flailing, chin rubs, head shaking, and active rejection of the infusate.

Whereas all rats were conditioned against intraoral sucrose infusions identically, a final test intraoral infusion was administered at three different intervals after the third pairing of sucrose and LiCl: 2 days, 3 months, and 6 months. All six rats tested after a 2-day interval rejected all the infused sucrose and did not gain weight during the test. After 3 months, three out of six rats gained $0.10-0.15$ grams during the 6.6- $\mathrm{ml}$ infusion; the mean intake, however, was not significantly different from the intakes of the 2-day rats. After 6 months, five out of six rats consumed from 0.3 to 2.0 grams of the 6.6- $\mathrm{ml}$ sucrose infusion. The mean intake of the 6-month rats was significantly greater than the intakes of the 2-day $(P<0.005)$ and the 3-month rats $(P<0.05)$. Both the 2 -day and 3 -month rats displayed aversive reactions to the sucrose infusions on the final test day. Although the 6-month rats also displayed aversive reactions, there appeared to be a brief latency of 1 or $2 \mathrm{~min}$ prior to the first active rejection responses in several of the rats. This difference in behavioral response between the groups was based on nonsystemic observations, however, and further work is required to measure these apparent differences.

\section{C-FLI IN THE NTS}

Intraoral infusions of $5 \%$ sucrose induced c-FLI in the NTS of 2-day and 3-month animals in a pattern consistent with our earlier observations (Figs. 2 and 3). c-FLI-positive cells were present in the caudal, intermediate, and rostral portions of the iNTS. The largest number of c-FLI-positive nuclei were found in the iNTS. The punctate nuclear staining was clustered in the medial part of the NTS; occasional cells were found just lateral to the fibers of solitary tract. In the caudal subpostremal NTS, stained cells were fewer in number and located in the ventral NTS just dorsal to the dorsal motor nucleus of the vagus. In the rostral NTS, c-FLI-positive cells were sparsely scattered ventral to the solitary tract with no evident clustering. No c-FLI-positive cells were observed in the AP adjacent to the NTS.

The distribution of c-FLI-positive cells was qualitatively identical in the NTS of 2-day and 3 -month rats. The mean number of c-FLI-positive cells per section was also very similar, although there were significantly more c-FLI-positive nuclei
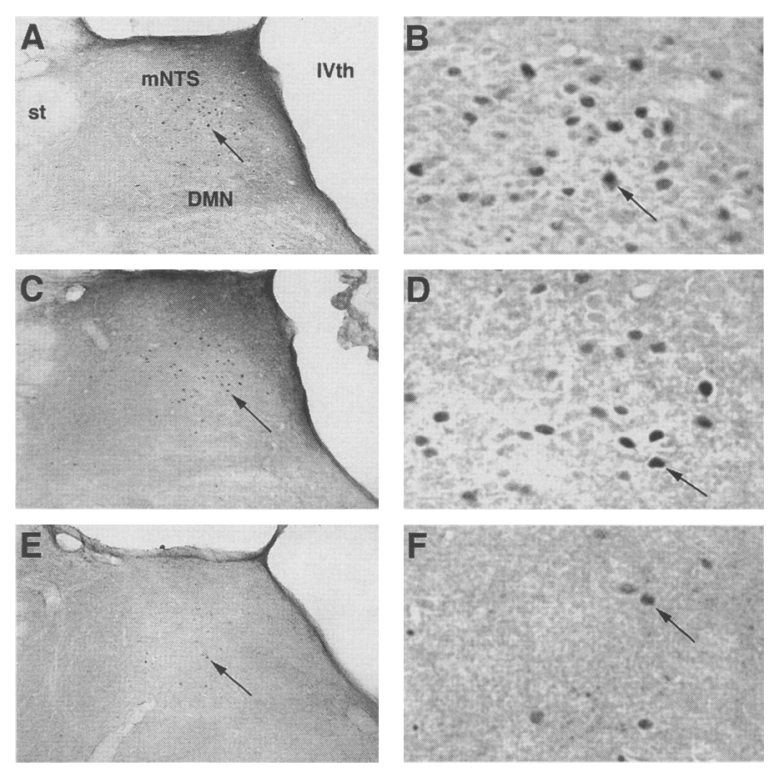

Figure 2: Examples of c-FLI in the iNTS of rats $1 \mathrm{hr}$ after intraoral infusions of $5 \%$ sucrose 2 days $(A, B), 3$ months $(C, D)$, and 6 months $(E, F)$ after third pairing of intraoral sucrose infusions and $\mathrm{LiCl}$. Arrows indicate individual c-FLI-positive nuclei seen at both low magnification $(40 \times$ in $A, C, E)$ and at higher magnification $(160 \times$ in $B, D, F)$. (st) Solitary tract; (mNTS) medial nucleus of the solitary tract; (DMN) dorsal motor nucleus of the vagus; (IV) fourth ventricle.

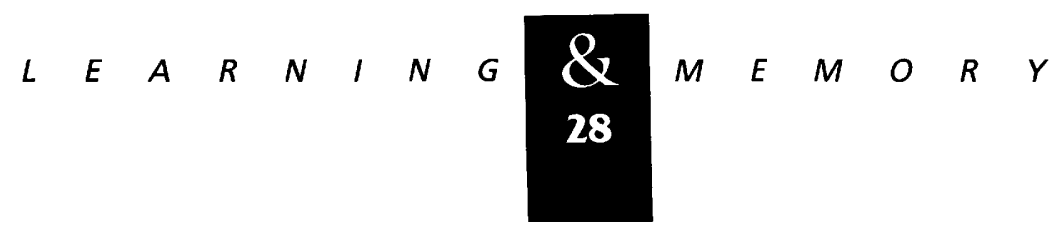




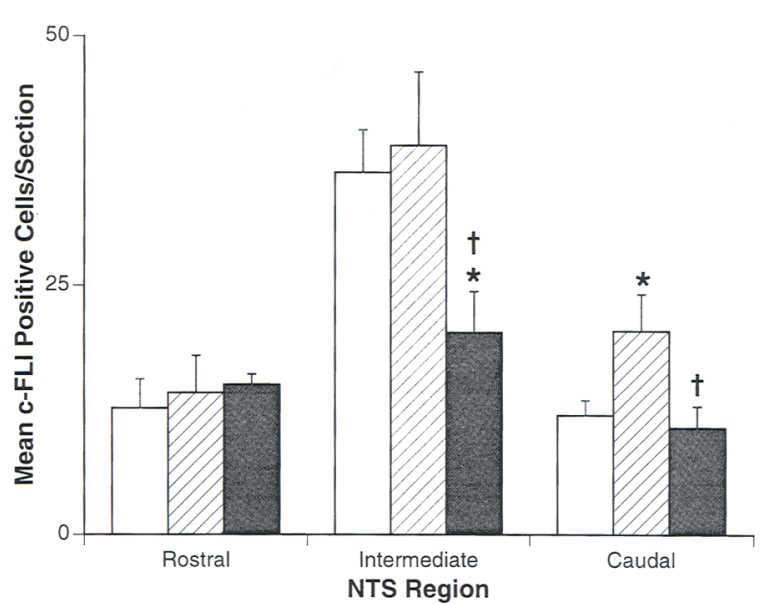

Figure 3: Quantification of C-FLI induced in the NTS of rats by intraoral $5 \%$ sucrose infusion $(6.6 \mathrm{ml}$ over 6 min) 2 days (open bars), 3 months (hatched bars), or 6 months (solid bars) after third pairing of sucrose with LiCl. (Asterisk) $P<0.05$ significantly different from 2-day group; (dagger) $P<0.05$ significantly different from 3 -month group. $n=6$ in each group.

in the caudal NTS of 3-month rats compared with 2-day rats. The pattern of c-FLI in the NTS of 6-month rats was also similar to that of the 2-day and 3-month rats. There were significantly fewer cells, however, in the intermediate NTS of 6-month rats compared with 2-day or 3-month rats $(P<0.05)$. There were also significantly fewer cells in the caudal NTS of 6-month rats compared with 3 -month rats $(P<0.05)$ but not to 2 -day rats.

\section{Discussion}

The CTA against sucrose persisted without attenuation for at least 3 months as evidenced by the almost complete rejection of intraorally infused sucrose 2 days and 3 months after acquisition. The CTA against sucrose, however, was significantly attenuated 6 months after the last pairing, because intake of intraoral sucrose increased significantly compared with 2 days and 3 months after acquisition. Because the rats received no exposure to sucrose (the conditioned taste) during these 6 months, the decrement in the behavioral expression of the CTA was attributable to partial forgetting of the CTA rather than extinction (Bouton 1994). Previous experiments have demonstrated retention of a CTA in rats for 20-60 days after a single pairing of a tastant and a toxic treatment (e.g., sodium chloride and $X$ ray; Garcia et al.
1972) or saccharin and $\mathrm{LiCl}$ (Martin and Timmins 1980; Steinert et al. 1980) and retention in monkeys of a CTA against almonds for 3 months (Matsuzawa et al. 1983). This is the first report on rats of a CTA persisting for 3-6 months after acquisition, and it confirms that CTA is an extremely enduring form of associative learning (Seligman 1970 ).

In addition to demonstrating both persistence and partial forgetting of CTA at the behavioral level, we also characterized alterations in the induction of c-FLI in the iNTS after sucrose infusions that have been demonstrated previously after acquisition of a CTA (Houpt et al. 1994; Swank and Bernstein 1994). The sucrose infusions administered 2 days and 3 months after acquisition induced equivalently high numbers of c-FLI-positive cells in the iNTS; however, an identical sucrose infusion 6 months after acquisition induced a significantly smaller number of c-FLI cells in the iNTS. Thus, the c-FLI response of the iNTS to sucrose parallels the behavioral expression of a CTA against sucrose 6 months after acquisition.

We draw two conclusions: (1) The altered sensitivity of the iNTS to sucrose infusions after CTA acquisition is extremely persistent. Previous papers by ourselves and others demonstrated c-FLI induction by sucrose and saccharin within days of the final acquisition trial (Houpt et al. 1994; Swank and Bernstein 1994). The current study demonstrates that c-FLI is still induced by an intraoral sucrose infusion 6 months after acquisition of a CTA against sucrose. No other putative neuronal correlates of CTA expression have been observed months after initial acquisition of the CTA. (2) The induction of c-FLI in the iNTS by an intraoral sucrose infusion is correlated with retention and forgetting of a CTA against sucrose as measured by sucrose intake during the infusion. The significant increase in intake of intraorally infused sucrose observed 6 months after CTA acquisition was accompanied by a significant decrease in the number of c-FLI-positive cells in the iNTS induced by the sucrose.

Thus, our attempts to dissociate the behavioral expression of a CTA and the induction of c-FLI in the iNTS by experimental extinction (Houpt et al. 1994) and spontaneous forgetting have failed. This strengthens the evidence that c-FLI in the iNTS is a neuronal correlate of the expression of a CTA against sucrose. Further experiments are required to determine whether c-FLI in these cells modulates transcription of tar-

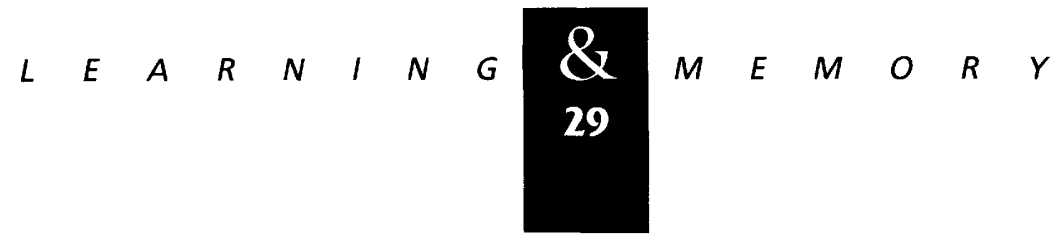


get genes involved in the neuronal plasticity that mediates CTA acquisition, extinction, and forgetting.

\section{Acknowledgments}

This work was supported by grants $\mathrm{MH} 15455$ and MH00149 (G.P.S.) and by the New York Obesity Center and the Whitehall Foundation (T.A.H.).

The publication costs of this article were defrayed in part by payment of page charges. This article must therefore be hereby marked "advertisement" in accordance with 18 USC section 1734 solely to indicate this fact.

\section{References}

Bouton, M.E. 1994. Conditioning, remembering, and forgetting. J. Exp. Psychol. 20: 219-231.

Garcia, J., B.K. McGowan, and K.F. Green. 1972. Biological constraints on conditioning. In Classical conditioning II: Current research and theory (ed. A.H. Black and W.F. Prokasy) pp. 21-43. Appleton-Century-Crofts, New York, NY.

Houpt, T.A., J.M. Philopena, T.C. Wessel, T.H. Joh, and G.P. Smith. 1994. Increased c-Fos expression in the rat nucleus of the solitary tract after conditioned taste aversion formation. Neurosci. Lett. 172: 1-5.

Martin, G.M. and W.K. Timmins. 1980. Taste-sickness associations in young rats over varying delays, stimulus, and test conditions. Anim. Learn. Behav. 8: 529-533.

Matsuzawa, T., Y. Hasegawa, S. Gotoh, and K. Wada. 1983. One-trial long-lasting food-aversion learning in wild Japanese monkeys (Macaca fuscata). Behav. Neural Biol. 39: 155-159.

Paxinos, G. and C. Watson. 1986. The rat brain in stereotaxic coordinates. Academic Press, San Diego, CA.

Seligman, M.E.P. 1970. On the generality of the laws of learning. Psychol. Rev. 77: 406-418.

Steinert, P.A., R.N. Infurna, and N.E. Spear. 1980. Long-term retention of a conditioned taste aversion in preweanling and adult rats. Anim. Learn. Behav. 8: 375-381.

Swank, M.W. and I.L. Bernstein. 1994. c-Fos induction in response to a conditioned stimulus after single trial taste aversion learning. Brain Res. 636: 202-208.

Tyrka, A. and G.P. Smith. 1993. SCH23390, but not raclopride, decreases intake of intraorally infused $10 \%$ sucrose in adult rats. Pharmacol. Biochem. Behav.

45: $243-246$.

Received April 3, 1996; accepted June 4, 1996.

$\begin{array}{llllllll}L & E & A & R & N & I & N & G \\ \mathbf{Q} \\ 30\end{array}$




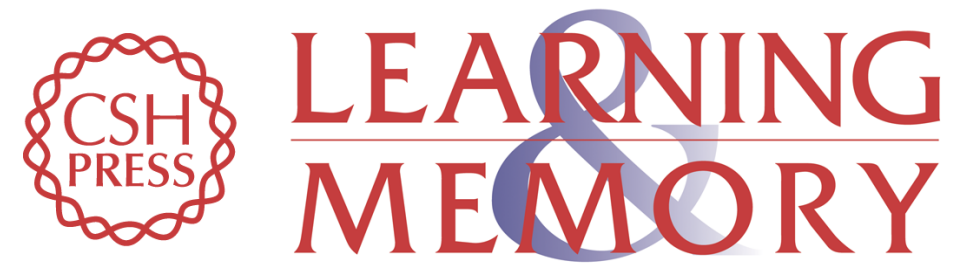

\section{c-Fos induction in the rat nucleus of the solitary tract correlates with the retention and forgetting of a conditioned taste aversion.}

T A Houpt, J M Philopena, T H Joh, et al.

Learn. Mem. 1996, 3:

Access the most recent version at doi:10.1101//m.3.1.25

\section{License}

Email Alerting

Receive free email alerts when new articles cite this article - sign up in the box at the Service top right corner of the article or click here. 